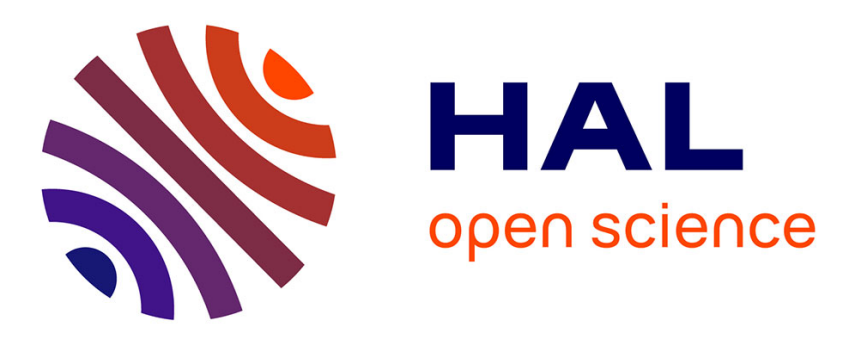

\title{
A Chance-Constrained Model \& Cutting Planes for Fixed Broadband Wireless Networks
}

\author{
Grit Classen, David Coudert, Arie Koster, Napoleao Nepomuceno
}

\section{To cite this version:}

Grit Classen, David Coudert, Arie Koster, Napoleao Nepomuceno. A Chance-Constrained Model \& Cutting Planes for Fixed Broadband Wireless Networks. Internationale Network Optimization Conference (INOC), Jun 2011, Hamburg, Germany. pp.37-42, 10.1007/978-3-642-21527-8_5 . inria00587669

\section{HAL Id: inria-00587669 \\ https://hal.inria.fr/inria-00587669}

Submitted on 21 Apr 2011

HAL is a multi-disciplinary open access archive for the deposit and dissemination of scientific research documents, whether they are published or not. The documents may come from teaching and research institutions in France or abroad, or from public or private research centers.
L'archive ouverte pluridisciplinaire HAL, est destinée au dépôt et à la diffusion de documents scientifiques de niveau recherche, publiés ou non, émanant des établissements d'enseignement et de recherche français ou étrangers, des laboratoires publics ou privés. 


\title{
A Chance-Constrained Model \& Cutting Planes for Fixed Broadband Wireless Networks *
}

\author{
Grit Claßen \\ Lehrstuhl II für Mathematik \\ RWTH Aachen University, 52056 Aachen, Germany \\ classen@math2.rwth-aachen.de \\ David Coudert \\ Mascotte, INRIA, I3S, CNRS, Université de Nice Sophia \\ Sophia Antipolis, France \\ david.coudert@inria.fr \\ Arie M. C. A. Koster \\ Lehrstuhl II für Mathematik \\ RWTH Aachen University, 52056 Aachen, Germany \\ koster@math2.rwth-aachen.de \\ Napoleão Nepomuceno \\ Institut for Matematik og Datalogi \\ Syddansk Universitet, Campusvej 55, DK-5230 Odense \\ napoleao@imada.sdu.dk
}

April 21, 2011

\begin{abstract}
In this paper, we propose a chance-constrained mathematical program for fixed broadband wireless networks under unreliable channel conditions. The model is reformulated as integer linear program and valid inequalities are derived for the corresponding polytope. Computational results show that by an exact separation approach the optimality gap is closed by $42 \%$ on average.
\end{abstract}

\section{Introduction}

Fixed broadband wireless (FBW) communications is a promising technology for delivering private highspeed data connections by means of microwave radio transmission [2]. Microwave, in the context of this work, refers to terrestrial point-to-point digital radio communications, usually employing highly directional antennas in clear line-of-sight and operating in licensed frequency bands. The rapid and relatively cheap deployment is especially interesting for emerging countries and remote locations as well as for private and isolated networks in urban areas (e.g., connected hospitals, parts of a harbour) where

* This paper results from a research cooperation which was promoted by the PROCOPE program, a bilateral program funded by the German Academic Exchange Service with funds of the BMBF and by the French Ministry of Foreign Affairs. Additionally, this work has been supported by ANR DIMAGREEN and ECOSCELLS, the PATHFINDER project, Région PACA, and SME 3ROAM as well as the excellence initiative of the German federal and state governments and by the UMIC Research Centre at RWTH Aachen University. The forth author gratefully acknowledges the financial support by the Villum Kann Rasmussen foundation. 
classical copper/fiber lines are too costly [9]. In contrast to wired networks, the capacity of a microwave link is not constant, but depends on the used modulation scheme, which in turn depends on the condition of the channel. Varying channel conditions result in varying link capacities.

In this paper, we extend our earlier study [3] of planning FBW networks under unreliable channel conditions. We restate a chance-constrained optimization model and, for the case where the outage probabilities are independent, an integer linear programming (ILP) formulation (Section 2). We generalize two classes of cutset-based valid inequalities (Section 3) and propose to separate them exactly. Preliminary computational results confirm the importance of these cuts (Section 4).

\section{Mathematical formulation}

The minimum cost design of a fixed broadband wireless network can be formulated as follows, cf. [4] for technical details. The network's topology is modeled as a digraph $G=(V, E)$ with $V$, the set of radio base stations and $E$, the set of directional microwave radio links. The traffic requirements are modeled by a set $K$. For each $k \in K, s^{k}$ denotes the origin, $t^{k}$ the destination, and $d^{k} \geq 0$ the expected demand.

For each microwave link $u v \in E$, the capacity is basically determined by the channel bandwidth (e.g., 7 $\mathrm{MHz}, 28 \mathrm{MHz}$ ) and the modulation scheme (e.g., 16-QAM, 128-QAM) used to transmit data. Where exactly one channel bandwidth has to be chosen at design stage, adaptive modulation is performed at runtime, depending on the channel conditions, i.e., if the receiving base station observes a deterioration in signal quality, the modulation scheme is lowered to avoid outage of the link.

Let $W_{u v}$ be the set of bandwidth choices available for arc $u v \in E$. The choice to operate link $u v \in E$ at bandwidth $b_{u v}^{w}, w \in W_{u v}$, implies a cost $c_{u v}^{w}$. The modulation scheme is modeled with a random variable $\eta_{u v}^{w}$ with (known) discrete probability, representing the number of bits per symbol of the current modulation scheme. The capacity of a microwave link is basically given by the product of $b_{u v}^{w}$ and $\eta_{u v}^{w}$.

Given an infeasibility tolerance $\epsilon>0$, our aim is to design a minimum cost network such that its capacity is sufficient with a probability of at least $1-\epsilon$. This joint chance constraint reads

$$
\mathcal{P}\left(\sum_{k \in K} d^{k} f_{u v}^{k} \leq \sum_{w \in W_{u v}} \eta_{u v}^{w} b_{u v}^{w} y_{u v}^{w} \quad \forall u v \in E\right) \geq 1-\epsilon
$$

with binary decision variables $y_{u v}^{w}$ indicating whether bandwidth $w \in W_{u v}$ is chosen for arc $u v \in E$ and flow variables $f_{u v}^{k}$ denoting the fraction of demand $d^{k}, k \in K$, routed on arc $u v \in E$.

For independent random variables $\eta_{u v}^{w}$, we can reformulate the left hand side of (1) as the product of probabilities by introducing the following notation: For arc $u v \in E$, let $M_{u v}^{w}$ be the set of modulations in case of bandwidth choice $w \in W_{u v}$ with, for $m \in M_{u v}^{w}, b_{u v}^{w m}$ the resulting capacity. Given $u v \in E$, $w \in W_{u v}$, and $m \in M_{u v}^{w}$, let $\rho_{u v}^{w m}$ be the probability that the link is operated at modulation $m$ or higher.

Now, we may assume that each link is operated at a chosen modulation (or higher) as long as the overall probability of the assumptions is at least $1-\epsilon$. For this, the binary decision variables $y$ obtain a new index $m$. The minimum cost fixed broadband wireless network design problem then reads:

$$
\begin{array}{cll}
\min & \sum_{u v \in E} \sum_{w \in W_{u v}} \sum_{m \in M_{u v}^{w}} c_{u v}^{w} y_{u v}^{w m} \\
\text { s.t. } & \sum_{u \in V: v u \in E} f_{v u}^{k}-\sum_{u \in V: u v \in E} f_{u v}^{k}= \begin{cases}1, & \text { if } v=s^{k}, \\
-1, & \text { if } v=t^{k}, \\
0, & \text { otherwise }\end{cases} & \\
& \sum_{w \in W_{u v}} \sum_{m \in M_{u v}^{w}} y_{u v}^{w m}=1 & \\
& \sum_{k \in K} d^{k} f_{u v}^{k} \leq \sum_{w \in W_{u v}} \sum_{m \in M_{u v}^{w}} b_{u v}^{w m} y_{u v}^{w m} & \\
& \prod_{u v \in E}\left(\sum_{w \in W_{u v}} \sum_{m \in M_{u v}^{w}} \rho_{u v}^{w m} y_{u v}^{w m}\right) \geq 1-\epsilon \in K \\
& f_{u v}^{k} \in[0,1], y_{u v}^{w m} \in\{0,1\} &
\end{array}
$$


Besides the total bandwidth cost function (2a) and the flow conservation constraints (2b), constraints (2c) ensure that exactly one bandwidth-modulation pair is chosen. Constraint (1) is now equivalently modeled in the link capacity constraints (2d) and in the solution confidence constraint (2e). (2d) ensure that all demands on one link can be fulfilled by the chosen bandwidth-modulation pair, whereas (2e) guarantees that the confidence of the solutions is at least $1-\epsilon$.

Note that we assume explicitly a hypothesis on the modulation scheme in constraints (2d). Obviously, for a given link and bandwidth, the lower the modulation scheme is, the lower the assumed capacity and the higher the probability that the effective capacity supports the routed traffic. In other words, more conservative hypotheses on the modulation schemes lead to more reliable solutions.

Constraint (2e) can be easily linearized: By employing monotonicity of logarithmic functions and because the logarithm of a product equals the sum of the logarithms, (2e) is equivalent to

$$
\sum_{u v \in E} \log \left(\sum_{w \in W_{u v}} \sum_{m \in M_{u v}^{w}} \rho_{u v}^{w m} y_{u v}^{w m}\right) \geq \log (1-\epsilon) .
$$

By constraints (2c), exactly one of the sum elements within each logarithmic function will be nonzero. Hence, (3) is equivalent to

$$
\sum_{u v \in E} \sum_{w \in W_{u v}} \sum_{m \in M_{u v}^{w}} \log \left(\rho_{u v}^{w m}\right) y_{u v}^{w m} \geq \log (1-\epsilon)
$$

\section{$3 \quad$ Valid inequalities}

Constraints (2b), (2c), and (2d) define a classical network design problem studied intensively in the literature, see [10] and the references therein. In particular, cut-based inequalities have been proven to be effective to enhance the performance of ILP solvers [1]. Let $S \subset V$ be a proper and nonempty subset of the nodes $V$ and $\bar{S}=V \backslash S$ its complement. The set $E(S, \bar{S}):=\{u v \in E: u \in S, v \in \bar{S}\}$, i.e., the set of arcs from $S$ to $\bar{S}$ defines a cutset. Similarly, let $K(S, \bar{S}):=\left\{k \in K: s^{k} \in S, t^{k} \in \bar{S}\right\}$ be the set of demands originating in $S$ and terminating in $\bar{S}$. Finally, let $d(S, \bar{S}):=\sum_{k \in K(S, \bar{S})} d^{k}$. An appropriate aggregation of constraints $(2 \mathrm{~b}),(2 \mathrm{~d})$, and nonnegativity of the variables results in the following base cutset inequality:

$$
\sum_{u v \in E(S, \bar{S})} \sum_{w \in W_{u v}} \sum_{m \in M_{u v}^{w}} b_{u v}^{w m} y_{u v}^{w m} \geq d(S, \bar{S})
$$

Chvátal-Gomory (CG) rounding yields two classes of valid inequalities.

Cutset Inequalities By dividing (5) by $a \in\left\{b_{u v}^{w m}: u v \in E(S, \bar{S}), w \in W_{u v}, m \in M_{u v}^{w}\right\}$ and rounding up both sides, the well-known cutset inequalities [10] are obtained:

$$
\sum_{u v \in E(S, \bar{S})} \sum_{w \in W_{u v}} \sum_{m \in M_{u v}^{w}}\left\lceil\frac{b_{u v}^{w m}}{a}\right\rceil y_{u v}^{w m} \geq\left\lceil\frac{d(S, \bar{S})}{a}\right\rceil
$$

Shifted Cutset Inequalities Instead of applying CG-rounding directly, we can first shift the coefficients of (5). Given a cutset $E(S, \bar{S})$, let $a_{u v}=\min _{w \in W_{u v}} \min _{m \in M_{u v}^{w}} b_{u v}^{w m}$ for $u v \in E(S, \bar{S})$. By (2c) and $a(S, \bar{S}):=\sum_{u v \in E(S, \bar{S})} a_{u v},(5)$ can be rewritten as:

$$
\sum_{u v \in E(S, \bar{S})} \sum_{w \in W_{u v}} \sum_{m \in M_{u v}^{w}}\left(b_{u v}^{w m}-a_{u v}\right) y_{u v}^{w m} \geq d(S, \bar{S})-a(S, \bar{S})
$$

Now, let $a^{\prime} \in\left\{b_{u v}^{w m}-a_{u v}: u v \in E(S, \bar{S}), w \in W_{u v}, m \in M_{u v}^{w}\right\}$. By CG-rounding, we obtain the following shifted cutset inequalities:

$$
\sum_{u v \in E(S, \bar{S})} \sum_{w \in W_{u v}} \sum_{m \in M_{u v}^{w}}\left\lceil\frac{b_{u v}^{w m}-a_{u v}}{a^{\prime}}\right\rceil y_{u v}^{w m} \geq\left\lceil\frac{d(S, \bar{S})-a(S, \bar{S})}{a^{\prime}}\right\rceil
$$

It can be shown that (6) and (8) define facets of the convex hull of feasible solutions under certain conditions (beyond the scope of this paper). 


\section{Computational Results}

Setting We have performed preliminary computational experiments on a $5 \times 5$ grid network $(|V|=25$, $|E|=80,|K|=50)$ based on [8]. We consider two bandwidth choices for each link: $7 \mathrm{MHz}(28 \mathrm{MHz})$ with cost 1000 (6000) using the 128-QAM (256-QAM) scheme, with an availability of 99.9\%. In fading conditions, these links will use the 16-QAM (32-QAM) scheme (with $100 \%$ availability).

By assuming the same availability for radio links using the highest modulation scheme and under the hypothesis that the lowest modulation scheme guarantees an availability of $100 \%$ (independent of the bandwidth), we can replace (4) by

$$
\sum_{u v \in E} \sum_{w \in W_{u v}} y_{u v}^{w 2} \leq\left\lfloor\frac{\log (1-\epsilon)}{\log (\rho)}\right\rfloor=: N
$$

where $\rho$ is the availability probability of the highest modulation scheme. Note that a larger infeasibility tolerance $\epsilon$ implies a larger value $N$, i.e., the reliability of the solutions decreases. We consider $N=$ $10(\epsilon=0.01), 20, \ldots, 80$ (no reliability).

All computations are performed with CPLEX 12.2 [6] on a Linux machine with $2.67 \mathrm{GHz}$ Intel Xeon X5650 processor and 12 GB RAM.

Optimality gap closed In this study, we limit ourselves to a comparison of the optimality gap with/without separation of violated cutset inequalities (6) and/or shifted cutset inequalities (8). To this end, the separation of these inequalities is done exactly by an auxiliary ILP (details omitted, cf. e.g., $[5,7])$. The cutset inequalities are separated only in the root node of the branch-and-bound tree.

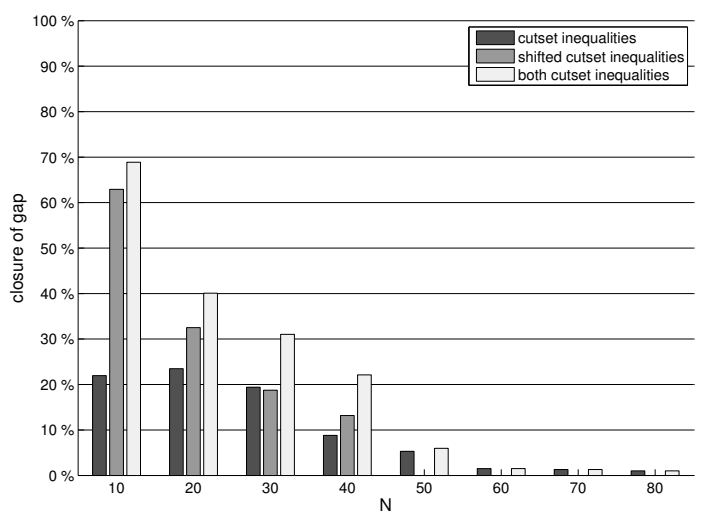

(a) Cutset inequalities only

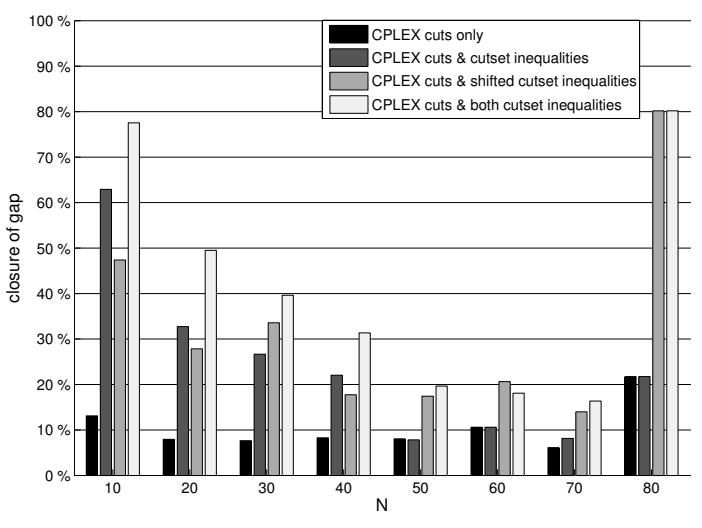

(b) CPLEX cuts and cutset inequalities

Figure 1: Optimality gap closed.

As a reference, we consider the optimality gap, i.e., the difference between LP relaxation and best known solution (computed by CPLEX with a time limit of $12 \mathrm{~h}$, optimal for $N=10,60,70,80$ ). Fig. 1 shows the optimality gap closed, i.e., the percental reduction of the optimality gap at the end of the root node. For the results in Fig.1(a), we disabled the internal cuts of CPLEX and separated (i) cutset inequalities (6), (ii) shifted cutset inequalities (8), and (iii) both. Inequalities (8) close the gap with $16 \%$ on average, whereas inequalities (6) close only $10 \%$. Obviously, the optimality gap is closed most by the combination of (6) and (8) (up to $69 \%$ for $N=10$ and $21 \%$ on average). With increasing $N$, the closure of the optimality gap decreases with hardly any closure from $N=60$. We conjecture that inequalities (6) and (8) are less likely violated since the constraints (2d) are less restrictive.

In Fig. 1(b), we enabled the internal cuts of CPLEX. The optimality gap closed by internal cuts is only $10 \%$ on average compared to $42 \%$ by the combination of internal cuts and cutset inequalities (6) and (8). Note that also CPLEX can separate cutset inequalities [1]: only for $N=80$ and (8), some multi-commodity flow (MCF) cuts are found. In case both types are separated, on average 54 violated inequalities are found (17 of type (6) and 37 of type (8)). Again, for increasing $N$ the optimality gap closed decreases, except for $N=80$ where $80 \%$ of the gap is closed (due to the MCF cuts). For $N=60$, 
the optimality gap is closed less by the combination of (6) and (8) than only by the shifted cutset inequalities (8). Such a phenomenon can occur due to varying internal CPLEX cuts.

\section{Concluding Remarks}

In this paper, we have presented a chance-constrained programming approach for the assignment of bandwidth in reliable fixed broadband wireless networks. We have proposed cutset inequalities and shifted cutset inequalities to enhance the computability of this problem. In our computational studies, we have discussed the optimality gap closed and compared the performance of the different cutset inequalities with and without internal CPLEX cuts. The results show that by the combination of the cutset and the shifted cutset inequalities, the optimality gap is closed by $41 \%$ on average if the internal cuts for CPLEX are enabled.

As future work, we intend to investigate more realistic network topologies, different probability models and the reliability regarding traffic fluctuations.

\section{References}

[1] Achterberg, T., Raack, C.: The MCF-separator: detecting and exploiting multi-commodity flow structures in MIPs. Mathematical Programming Computation 2, 125-165 (2010)

[2] Anderson, H.: Fixed Broadband Wireless System Design, first edn. John Wiley \& Sons (2003)

[3] Claßen, G., Coudert, D., Koster, A., Nepomuceno, N.: Bandwidth assignment for reliable fixed broadband wireless networks. In: IEEE WoWMoM 2011. IEEE, Lucca, Italy (2011)

[4] Coudert, D., Nepomuceno, N., Rivano, H.: Power-efficient radio configuration in fixed broadband wireless networks. Computer Communications 33(8), 898-906 (2010)

[5] Fischetti, M., Lodi, A., Salvagnin, D.: Just MIP it! Ann. Info. Systems 10, 39-70 (2009)

[6] IBM - ILOG: CPLEX Optimization Studio 12.2. URL http://www.ilog.com/products/cplex

[7] Koster, A.M.C.A., Kutschka, M., Raack, C.: Cutset inequalities for robust network design. In: International Network Optimization Conference, INOC 2011 (2011)

[8] Larsson, T., Yuan, D.: An augmented lagrangian algorithm for large scale multicommodity routing. Computational Optimization and Applications 27(2), 187-215 (2004)

[9] Lehpamer, H.: Microwave transmission networks: planning, design, and deployment. McGraw-Hill (2010)

[10] Raack, C., Koster, A.M.C.A., Orlowski, S., Wessäly, R.: On cut-based inequalities for capacitated network design polyhedra. Networks 57, 141-156 (2011) 